\title{
SHELL STRESS ANALYSIS USING A VARIATIONAL METHOD BASED ON THREE-DIMENSIONAL FUNCTIONS WITH FINITE CARRIERS
}

\author{
Ali Kamil Jebur ${ }^{1}$, Enaam Obeid Hassoun ${ }^{1}$, Hussain Abdulaziz Abrahem ${ }^{1}$, Khayrullin Farid Sagitovich ${ }^{2}$, \\ Sakhbiev Oleg Mirgasimovich ${ }^{2}$ \\ ${ }^{1}$ University of Technology, Iraq \\ ${ }^{2}$ Kazan National Research Technological University, Kazan, Russia
}

A modification of the method is presented, which allows one to analyze a thin shell calculation by specifying the geometry only in the Cartesian coordinate system. A variational method is presented for determining the stressstrain state of three-dimensional elastic structures based on the use of approximating functions with finite carriers of an arbitrary degree of approximation. The presented method can be successfully used both for the calculation of three-dimensional composite structures and for the calculation of thin shells using curvilinear coordinate systems, a comparison between the modeling of equation programming with the Ansys software gives a good indication for corrections of the method modification.

Key words: variational method, three-dimensional elastic structures, thin shells, deformation, Ansys software

\section{INTRODUCTION}

The Global Cartesian coordinate system is introduced $\widetilde{x}$, $\widetilde{y}, \widetilde{z}$, in which the investigated three-dimensional structure is divided into subdomains in the form of curved hexagons $V_{k}$ (Fig.1). Hex faces are assumed to be piecewise smooth surfaces $\left(\Omega_{i}, i=\overline{1,6}\right)$. A local Cartesian coordinate system is introduced $x, y, z$, associated with a structural element. Note that instead of the local Cartesian coordinate system, $x, y, z$, a curvilinear coordinate system can be chosen, which is considered in the work [1].

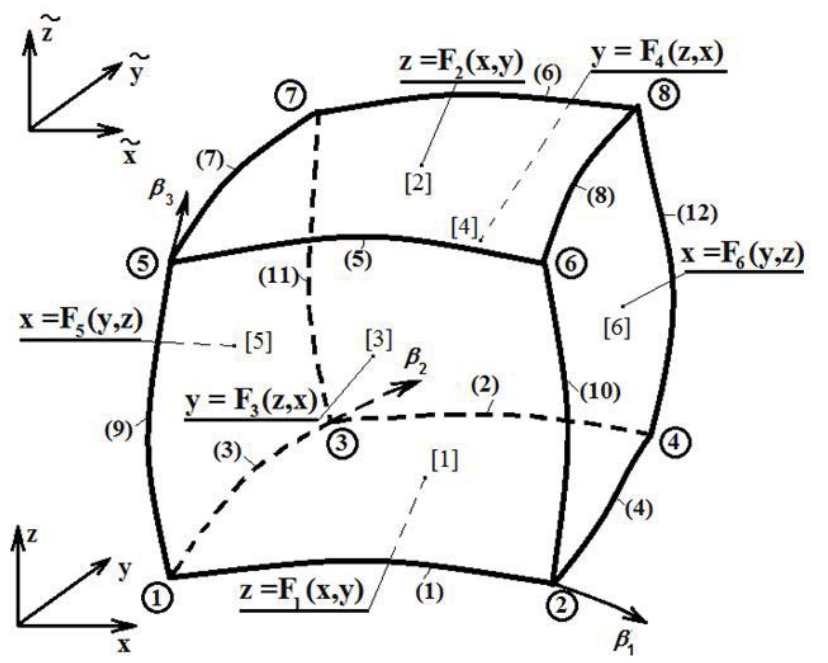

Figure 1: three-dimensional structure

The local coordinate system, $x, y, z$, is chosen so that the equations of the faces can be set relative to the corresponding coordinate planes in the following form:

$$
\begin{aligned}
& z=F_{1}(x, y), \quad z=F_{2}(x, y), y=F_{3}(z, x), y=F_{4}(z, x) \\
& x=F_{5}(y, z), x=F_{6}(y, z)
\end{aligned}
$$

Where $F i, i=\overline{1,6}$ - unambiguous class functions $C^{1}$. The figure shows the numbers of the sides in parentheses; in circles, the numbers of the corner points (nodes) of the sub-region; and in squares, the numbers of the faces $\Omega_{i}$. Within subarea $V_{k}$, a curvilinear coordinate system is introduced, $\beta_{1}, \beta_{2}, \beta_{3}$, which is related to the coordinate system, $x, y, z$, in the following way:

$$
\begin{aligned}
& x=F_{5}\left(y_{5}, z_{5}\right)\left(1-\beta_{1}\right)+F_{6}\left(y_{6}, z_{6}\right) \beta_{1}+d_{1}\left(\beta_{1}, \beta_{2}, \beta_{3}\right), \\
& y=F_{3}\left(z_{3}, x_{3}\right)\left(1-\beta_{2}\right)+F_{4}\left(z_{4}, x_{4}\right) \beta_{2}+d_{2}\left(\beta_{1}, \beta_{2}, \beta_{3}\right), \\
& z=F_{1}\left(x_{1}, y_{1}\right)\left(1-\beta_{3}\right)+F_{2}\left(x_{2}, y_{2}\right) \beta_{3}+d_{3}\left(\beta_{1}, \beta_{2}, \beta_{3}\right),
\end{aligned}
$$

where the functions, $y_{5}=y_{5}\left(\beta_{2}, \beta_{3}\right), z_{5}=z_{5}\left(\beta_{2}, \beta_{3}\right), d_{1}$ etc., are selected in this way [2], what's on the verge $\Omega_{i}{ }^{k}, i=\overline{1,6}$ sub-regions $V_{k}$ equations (2) go into the equations of these faces, on the boundary lines $\lambda_{i}^{k}, j=\overline{1,12}$ - into the equations of these lines; $0 \leq \beta_{1}, \beta_{2}, \beta_{3} \leq 1$. Moreover, the coordinate system on these lines, $\beta_{1}, \beta_{2}, \beta_{3}$, forms a uniform coordinate grid. As a result, if two sub domains are joined along a certain face and if this face in these subdomains is defined by the same equation, then the local coordinate grid on it for these subdomains will be the same. This ensures the continuity of the desired functions during transition from one subdomain to another. It also makes it easy to fulfill the geometric boundary conditions and those meant for joining the desired functions at the boundaries of the subdomains $V_{k}$.

Displacements defined in the global coordinate system $\widetilde{x}, \widetilde{y}, \widetilde{z}$, within the subdomain are represented as:

$\widetilde{u}_{i}=\sum_{n=1}^{N} \sum_{m=1}^{M} \sum_{l=1}^{L} D_{i n m l}^{k} t_{n}\left(\beta_{1}\right) t_{m}\left(\beta_{2}\right) t_{l}\left(\beta_{3}\right)$

$D^{k}{ }^{k}{ }^{n+1}-$ Unknown constants; form functions

$t_{1}\left(\beta_{1}\right)=1-\beta_{1}, t_{2}\left(\beta_{1}\right)=\beta_{1}$

$t_{m}\left(\beta_{1}\right)=t_{1}\left(\beta_{1}\right)\left[t_{2}\left(\beta_{1}\right)\right]^{m-2}(m=\overline{3, M})$ 
Note that only part of the unknown have physical meaning, namely they are nodal displacements of a subdomain $V_{k}$.

The triple sum (3) is written in matrix form:

$$
\{\tilde{u}\}=\left[D^{k}\right]\{t\}
$$

Where $\{\tilde{u}\}^{T}=\left\{\tilde{u}_{1}, \tilde{u}_{2}, \tilde{u}_{3}\right\},\{t\}=\left\{t_{1}, t_{2}, \ldots, t_{J}\right\}$. form functions defined in the coordinate system $\beta_{1}, \beta_{2}, \beta_{3}, ;\left[D^{k}\right]$ - matrix of unknown constants of dimension $3 \times J, J=N \cdot M \cdot L$.

Displacements in a local curvilinear coordinate system, $\beta_{1}, \beta_{2}, \beta_{3}$, are recorded through movements in the global Cartesian coordinate system, $\widetilde{x}, \widetilde{y}, \widetilde{z}$.

$\left.\left.\left\{\mu^{\beta^{k}}\right\}=\mid C^{\beta^{k}}\right] \cdot \mid C^{k}\right] \cdot\{\tilde{u}\}$

Where $\left|C^{\beta^{k}}\right|$. matrix of guide cosines of a local curv linear coordinate system, $\beta_{1}, \beta_{2}, \beta_{3}$ in the local Cartesian coordinate system, $x, y, z,\left[C^{k}\right]-$ matrix of guide cosines of the local Cartesian coordinate svstem. $x, y, z$, in the global cartesian coordinate system $\widetilde{x}, \widetilde{y}, \widetilde{z}$.

A class of problems is considered in the displacements and strains that are small; further, Hooke's law is valid. Substituting the approximating functions (4) into relations (5), then into the formula to determine the strains in a curvilinear coordinate system, using Hooke's law for the bulk stress state and the well-known formulas for the potential strain energy [4], we obtain the formula for the potential strain energy of the sub-region $V_{k}$ :

$\Pi_{k}=\frac{1}{2} \int_{V^{k}}\left\{t^{k}\right\}^{T}\left[D^{k}\right]^{\gamma}\left[C^{k}\right]^{T}\left[C^{\beta^{k}}\right]^{r}\left[A^{k}\right]^{r}[E] \times$

$\times\left[A^{k}\right]\left[C^{\beta^{k}}\left[C^{k}\right]\left[D^{k}\right]\left\{t^{k}\right\} d V\right.$

Where $[E]$ - elasticity matrix defined by known relations $[3],\left[A^{k}\right]$ - differential matrix, which has the following form:

$$
\left[A^{k}\right]=\left[\begin{array}{ccc}
\frac{1}{A_{1}} \frac{\partial}{\partial \beta_{1}} & , k_{12}, & k_{13} \\
k_{21} & , \frac{1}{A_{2}} \frac{\partial}{\partial \beta_{2}}, & k_{23} \\
k_{31} & , k_{32}, & \frac{1}{A_{3}} \frac{\partial}{\partial \beta_{3}} \\
\frac{1}{A_{2}} \frac{\partial}{\partial \beta_{2}}-k_{12} & , \frac{1}{A_{1}} \frac{\partial}{\partial \beta_{1}}-k_{21}, & 0 \\
0 & , \frac{1}{A_{3}} \frac{\partial}{\partial \beta_{3}}-k_{23}, & \frac{1}{A_{2}} \frac{\partial}{\partial \beta_{2}}-k_{32} \\
\frac{1}{A_{3}} \frac{\partial}{\partial \beta_{3}}-k_{13} & , 0, & \frac{1}{A_{1}} \frac{\partial}{\partial \beta_{1}}-k_{31}
\end{array}\right]
$$

$A_{i}=\sqrt{\sum_{j=1}^{3}\left(\frac{\partial x_{j}}{\partial \beta_{i}}\right)^{2}}$

$k_{i j}=\frac{1}{A_{i} A_{j}} \frac{\partial A_{i}}{\partial \beta_{j}}$

To determine the stress-strain state of the structure, the Lagrange variation principle [5] is used, on the basis of which the condition should be satisfied:

$\delta E=\sum_{k=1}^{K} \delta E_{k}=\sum_{k=1}^{K} \delta \Pi_{k}-\delta W_{k}=0$
Where $E$ - total design energy, $E_{k}$ - total energy subdomain $V_{k}, \Pi_{k}$ - potential strain energy, which is calculated in an orthogonal curvilinear coordinate system, $\beta_{1}, \beta_{2}, \beta_{3}$, $\delta W_{k}$ - variation of the work of external forces of a subdomain $V_{k}, K$ - number of subdomains.

From variation equation (7), we obtain a system of equations to determine unknown constants $D^{k}{ }_{i n m l}$.

\section{METHOD IMPLEMENTATION}

The distinctive features of the presented methodology are as follows: arbitrary order of approximation of the desired functions; integration in a curved coordinate system, $\beta_{1}, \beta_{2}, \beta_{3}$, which makes the integration procedure the same for any body shape; there is no need to describe the geometry of objects in a curvilinear system, $\beta_{1}, \beta_{2}, \beta_{3}$, since this system is internal and is constructed algorithmically based on the Cartesian coordinate system, $x, y, z$.

Using the presented method, a thin cylindrical shell clamped at the ends (Fig. 2) was calculated $L=50 \mathrm{~cm}$, loaded with an internal pressure of intensity $q=24 \mathrm{MPa}$. Cylinder inner radius $\mathrm{R}=50 \mathrm{~cm}$, cylinder wall thickness $\mathrm{t}=5 \mathrm{~cm}$, elastic modulus $\mathrm{E}=200 \mathrm{GPa}$, Poisson's ratio $v=0.3$. Due to the presence of symmetry planes, oneeighth of the shell was considered, which was limited to the coordinate planes, $x, y, z$. In the calculation, two subdomains (two finite elements) were used.

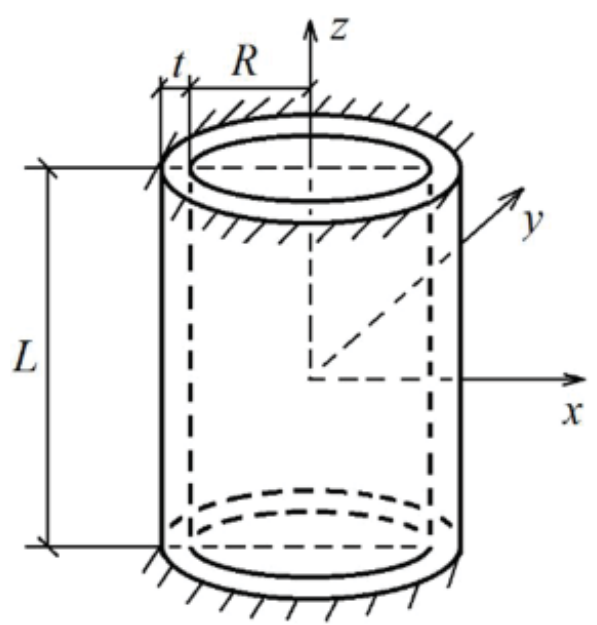

Figure 2

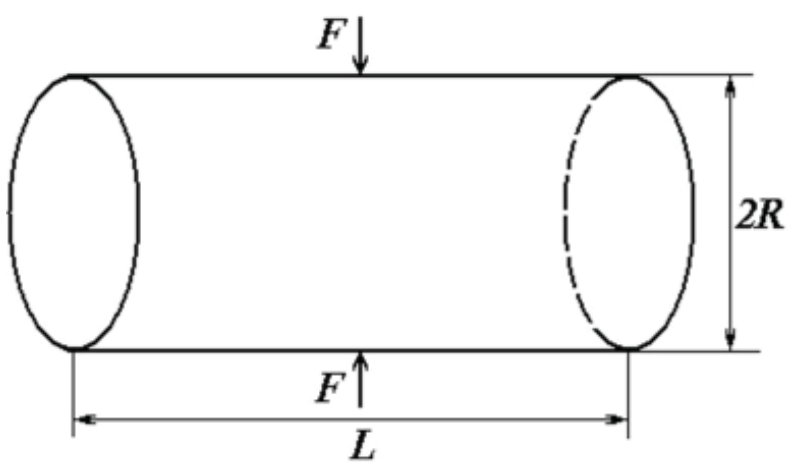

Figure 3 
Table 1: Stresses in a clamped shell

\begin{tabular}{|c|c|c|c|c|c|c|c|c|c|}
\hline Coordinates of & \multicolumn{7}{|c|}{$N=M=L, \mathrm{MPa}$} & \multicolumn{2}{|c|}{ [6], MPa } \\
\hline & & \multicolumn{2}{|c|}{4} & \multicolumn{2}{|c|}{6} & \multicolumn{2}{|c|}{8} & & \\
\hline$x$ & $z$ & $\sigma_{77}$ & $\sigma_{w y}$ & $\sigma_{77}$ & $\sigma_{v y}$ & $\sigma_{77}$ & $\sigma_{v w}$ & $\sigma_{77}$ & $\sigma_{v w}$ \\
\hline 50 & 0 & -178.8 & 143.6 & -132 & 156.9 & -120.7 & 162.7 & -113.2 & 176.1 \\
\hline 55 & 0 & 217.4 & 266.3 & 191.4 & 256.3 & 184 & 252.9 & 175.3 & 242.4 \\
\hline 50 & 8,33 & -69.1 & 150.6 & -94 & 139.3 & -78.6 & 153.1 & -84.3 & 148.0 \\
\hline 55 & 8,33 & 139.9 & 192.6 & 154.8 & 207.2 & 143.7 & 197.7 & 147.7 & 201.6 \\
\hline 50 & 25 & 447.7 & 191.9 & 550.4 & 235.9 & 599.5 & 256.9 & 591.9 & 253.7 \\
\hline 55 & 25 & -340.2 & -145.8 & -440.7 & -188.9 & -468.4 & -200.7 & -449.5 & -192.6 \\
\hline
\end{tabular}

\section{RESULT AND DISCUSSIONS}

The calculation results are shown in Table 1 , where stress values are given. $\sigma_{z z}$ and $\sigma_{y y}$ are at points with coordinates $(x, 0, z)$. The last two columns present the results from [6], in which this construction was divided into 300 elements.

For a fairly thin cylindrical shell with free ends under the action of a self-balanced system of two concentrated forces (Fig. 3), Table 2 compares the results obtained by this method with the results of other authors. The results are given for the following numerical parameters: $L=26.2$ $\mathrm{cm} ; \mathrm{R}=12.5 \mathrm{~cm} ; \mathrm{R} / \mathrm{t}=52.5 ; \mathrm{F}=453 \mathrm{~N} ; \mathrm{E}=74 \mathrm{GPa}$; $v=0.3125 ; R$ is the radius of the median surface. The calculation was carried out by two finite elements using symmetry (1/8 of the shell). Here are the values of the maximum deflection wmax for various values of the orders of the approximating functions. In [7], a solution was obtained for an inextensible shell, on the basis of which $\mathrm{w}_{\text {max }}=0.275 \mathrm{~cm}$.

Table 2: Shell deflections (cm)

\begin{tabular}{|c|c|c|c|c|}
\hline $\begin{array}{c}\text { Finiteelement } \\
\text { mesh }\end{array}$ & [8] & [9] & $\mathrm{N}=\mathrm{M}=\mathrm{L}=6$ & $\mathrm{~N}=\mathrm{M}=\mathrm{L}=8$ \\
\hline $1 \times 1$ & 0.264 & 0.269 & \multirow{5}{*}{0.284} & \multirow{5}{*}{0.285} \\
\hline $2 \times 2$ & 0.280 & 0.284 & & \\
\hline $4 \times 4$ & 0.287 & 0.288 & & \\
\hline $6 \times 6$ & 0.288 & - & & \\
\hline $8 \times 8$ & 0.289 & - & & \\
\hline
\end{tabular}
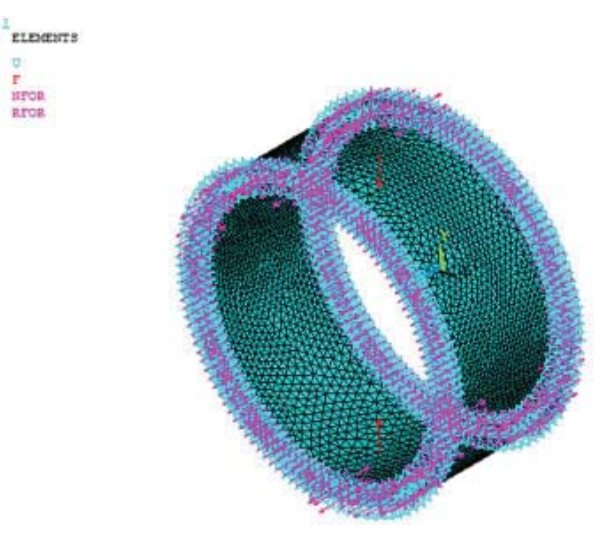

Figure 4: fixed-fixed supported by ANSYS

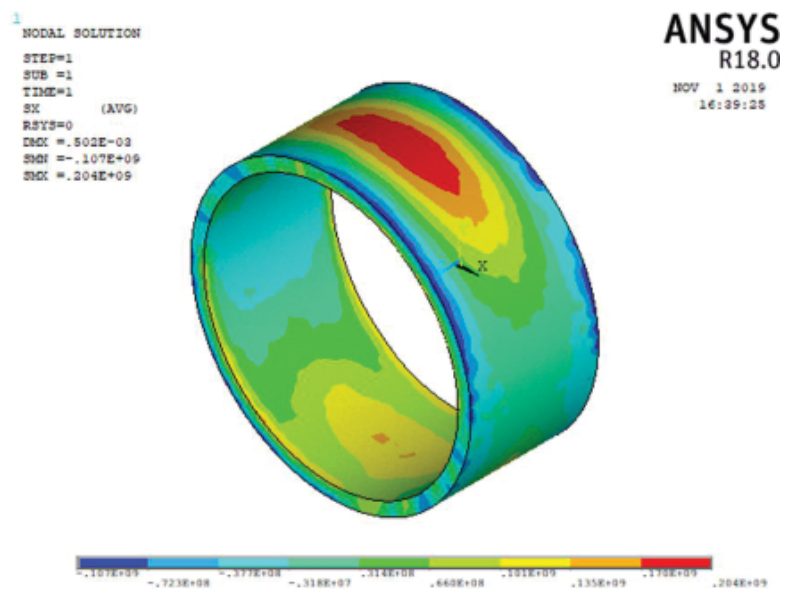

Figure 5: analysis by ANSYS fixed-fixed supported case 1 etemers

ANSYS

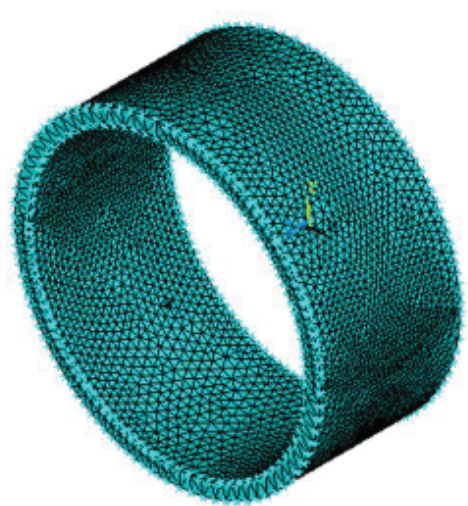

Figure 6: free-free supported by ANSYS

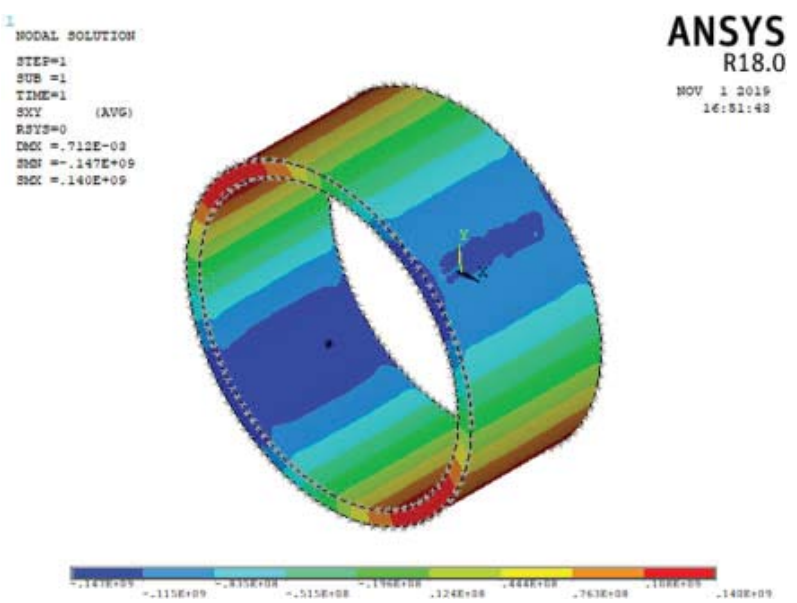

Figure 7: free-free supported by ANSYS 

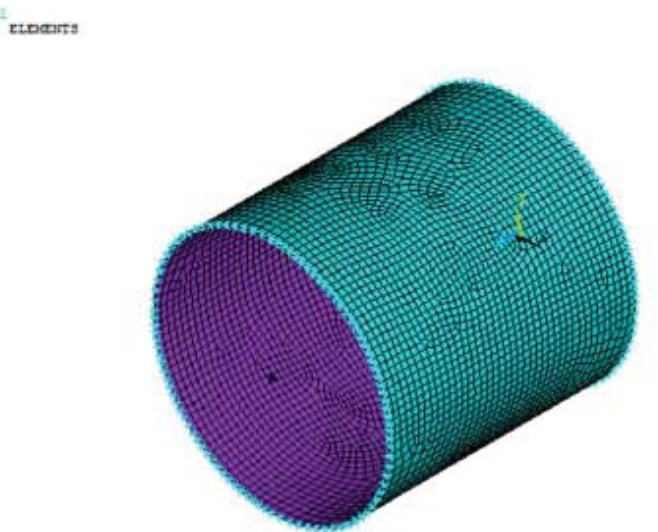

Figure 8: case 2 analysis by ANSYS

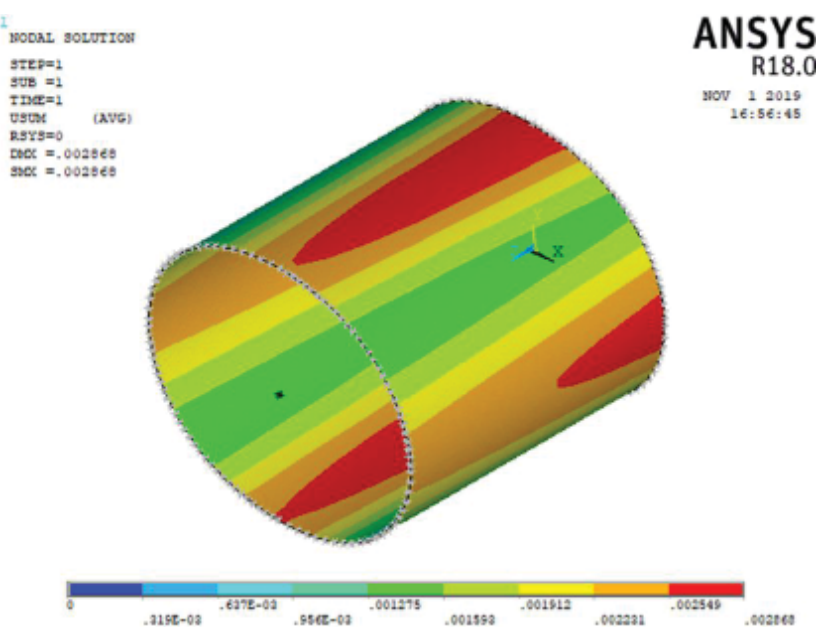

Figure 9: max deflection analysis by ANSYS case 2

The using of the Ansys software for analysis and comparison for case 1 and 2, Fig. 4 shows the auto-meshing that gave deformations in coordinates $50,8.33$ up to $0.5 \mathrm{~cm}$ in fixed-fixed in Fig. 5 and $0.7 \mathrm{~cm}$ in free-free in Fig. 7. For case 2, the deformation result shows in Fig. 9 up to $0.28 \mathrm{~cm}$ compatible with the deformation analysis in Table 2; finite elements mesh are more than $4 \times 4$ which indicates more accuracy when the fine mesh is done in the Ansys software.

\section{CONCLUSIONS}

As can be seen from the calculation results, the presented method agrees very well with the results of calculations by other authors, in which a significantly larger number of elements were used. Thus, the considered method allows one, describing the geometry of shells only in the Cartesian coordinate system, to determine the stress-strain of thin shells. Moreover, to obtain acceptable results, it is not necessary to split the structure into a large number of elements.

\section{REFERENCES}

1. Khayrullin F.S., Sakhbiev O.M. The calculation of orthotropic structures by the variational method based on three-dimensional functions with finite carriers // Bulletin of the Perm National Research Polytechnic University. Mechanics. - 2017. No. 2 - S. 195-207.
2. Khayrullin F.S., Sakhbiev O.M. On the method of calculating three-dimensional structures of complex shape // Bulletin of Kazan Technological University, 2014, v.17, No. 23, S.328-330.

3. Sakhbiev O.M., Khayrullin F.S. Modeling of deformations of thin shells based on three-dimensional functions with finite carriers // Scientific progress - creativity of young people: Materials of the XII international youth scientific conference on natural sciences and technical disciplines. Part. 1. - Yoshkar-Ola. PSTU, 2017 - S. 127-129.

4. Novozhilov V.V. Theory of elasticity. $-\mathrm{L}$.: Sudpromgiz, 1958, $371 \mathrm{p}$.

5. Abovsky N. P., Andreev N. P., Deruga A. P. Variational principles of the theory of elasticity and theory of shells. - M .: Nauka, 1978.- 288 p

6. Gureeva N.A. Hybrid finite element of loaded bodies. Izv. universities. North Cav. region. Ser. Technical science. - No. 1. - 2007. - p. 31-33.

7. Timoshenko SP, Voinovsky-Krieger S. Plates and shells. - M .: Nauka, 1966 .-- 636 p.

8. Ashwell D. G., Sabir A. B. A new cylindrical shell finite elements based on simple independent strain function // International Journal of Mechanical Sciences. - 1972. - V. 14. - No. 3. - P. 171 - 183.

9. Dawe D. J. High-order triangular finite element for shells analysis // International Journal of Solids and Structures. - 1975. - V. 11 - No. 10. - P. 1097 - 1110
Paper submitted: 22.11.2019.

Paper accepted: 16.02.2020.

This is an open access article distributed under the CC $B Y-N C-N D 4.0$ terms and conditions. 